\title{
Telerehabilitation for Rural Veterans: A Qualitative Assessment of Barriers and Facilitators to Implementation
}

This article was published in the following Dove Press journal: Journal of Multidisciplinary Healthcare

\author{
Jennifer L Hale-Gallardo' \\ Consuelo $M$ Kreider $\mathbb{D D}^{2,3}$ \\ Huanguang Jia (1D) \\ Gail Castaneda $\mathbb{D}^{2}$ \\ I Magaly Freytes $\mathbb{D}^{\prime}$ \\ Diane C Cowper Ripley ${ }^{2}$ \\ Zaccheus J Ahonle $\mathbb{D}^{3}$ \\ Kimberly Findley' \\ Sergio Romero (iD) ${ }^{1,2}$
}

'Department of Veterans Affairs, North Florida/South Georgia Veterans Health System, Gainesville, Florida, USA;

${ }^{2}$ Department of Veterans Affairs, Veterans Rural Health Resource CenterGainesville, Office of Rural Health, Gainesville, Florida, USA; ${ }^{3}$ Department of Occupational Therapy, University of Florida, Gainesville, Florida, USA
Correspondence: Sergio Romero Veterans Rural Health Resource Center Gainesville, North Florida/South Georgia Veterans Health System, I60I S.W. Archer Road, Gainesville, Florida 32608, USA

Tel +I 352-264-3845

Email Sergio.Romero@va.gov
Purpose: Telerehabilitation (TR) is increasingly being used to meet the rehabilitation needs of individuals living in rural areas. Nevertheless, reports on TR implementation for rural patients remain limited. As part of a broader evaluation, this study investigated barriers and facilitators to the implementation of a national TR program to meet the needs of rural Veterans Health Administration (VHA) patients.

Methods: This study applied a qualitative approach to the RE-AIM framework to investigate barriers and facilitators impacting TR implementation. We conducted in-depth, semistructured interviews with ten program managers and medical directors within the VHA at three time points during the first 18 months of implementation. Interviews were analyzed using thematic analysis.

Results: Three themes were identified describing key cultural, infrastructural and logistical, and environmental barriers impacting the reach, adoption, and implementation of TR. Within the themes, facilitators for TR were also identified to include, allowing providers flexibility in implementing TR, mentorship and development of creative approaches to TR training, overcoming infrastructural and logistical TR barriers through championing, and continuous sharing of lessons learned in a community of practice.

Discussion: This study explicates salient barriers and facilitators encountered during the first 18 months of implementation of a TR program within a national healthcare system in the United States. Implementing TR to meet the rehabilitation needs of Veterans in resource-limited rural environments requires creative approaches and flexibility, as well as perseverance and consistent championing in order to overcome cultural challenges. This, in combination with infrastructural challenges, such as lack of broadband, adds greater complexity to meeting the needs of rural patients. This study provides new and in-depth understanding of the processes by which TR is implemented in a large healthcare system and points to practical real-world lessons in implementing TR for rural patients.

Keywords: telemedicine, health, culture, technology, evaluation, implementation science

\section{Introduction}

Rehabilitation enhances the recovery of patients who suffer physical, cognitive, or emotional trauma by improving their health and quality of life. For those with mobility restrictions or who live in rural areas, however, rehabilitation is not always accessible. ${ }^{1-4}$ In a study examining travel distances to healthcare for Veterans in the United States, over half of the enrollees in the Veterans Health Administration (VHA) traveled more than 25 miles $^{5}$ a majority of Veteran enrollees $(58 \%)$ reside in rural areas. ${ }^{6}$ For rural Veterans with disabilities who may face physical, 
cognitive, or emotional obstacles to access needed rehabilitation, geographic barriers and the multiple visits frequently required, further magnify the challenge. ${ }^{7}$

The VHA has been a forerunner in implementing telehealth - the use of communication technologies to deliver clinical services and healthcare remotely. ${ }^{8-11}$ In recent decades, the VHA has expanded its telehealth services to include telerehabilitation (TR), a promising solution for rural Veterans in need of rehabilitation to regain optimal functioning. ${ }^{12-16}$ Reports on TR show it offers advantages over face-to-face rehabilitation by overcoming obstacles of travel distance and time and expanding health system capacity. ${ }^{4,17-22}$

Implementing TR in a way that is satisfactory to both providers and patients is a complex process. ${ }^{23,24}$ In the last decade, extending the traditional model of point-to-point telecare to include newer technologies, such as smartphones and internet-based telecommunications tools, has required the integration and interoperability of technologies within dynamic and rapidly evolving healthcare contexts. ${ }^{25}$ When these technologies are applied to rehabilitation within a large healthcare system, the complexity increases. ${ }^{26}$ Moreover, these complexities are compounded when extended to providing telerehabilitation to rural and highly rural areas. ${ }^{27,28}$ As part of a broader evaluation, we investigated barriers and facilitators to the implementation of a VHA-wide TR program for rural Veterans.

\section{Background on TR-EWI}

The Telerehabilitation Enterprise-Wide Initiative (TR-EWI) was initiated in 2017 by the VHA's Office of Rural Health (ORH) in partnership with the program office of Physical Medicine and Rehabilitation (PMR). The goal was to expand the delivery of rehabilitation services by leveraging centrally located, specialized rehabilitation expertise at "Hubs" and extending this expertise to "Spokes." These Spokes included rural VHA facilities and CommunityBased Outpatient Clinics (CBOCs) that provide services to rural Veterans who otherwise lack access to main VA facilities. Preliminary analysis of patient utilization data suggests that the TR-EWI program has supported dramatic expansion of TR throughout VHA-achieving an $80.2 \%$ increase in the number of rural Veterans who received TR during fiscal years (FY) 2017 and 2018. ${ }^{29}$

Three Polytrauma Rehabilitation Centers (Richmond, San Antonio and Minneapolis) and one Polytrauma Network Site (Seattle) were selected and have been operating as Hub sites for TR-EWI. Approved Spokes who applied to expand TR for their patients were assigned to a Hub primarily based on geographic proximity. Each Hub was allotted funds to hire a physician, a program manager, two rehabilitation therapists, a program assistant, and a telehealth technician. Each Spoke was allocated funds to hire a rehabilitation therapist and telehealth technician. As all Spokes were in rural areas, the rehabilitation specialist at each Spoke was hired to increase access to TR for rural Veteran patients. Hub sites have the expertise of providing a broad array of rehabilitation therapies and have provided mentorship and training to Spokes on the therapeutic protocols needed most by their rural patients. At the same time, Hubs meet additional rehabilitation needs of Veteran patients at Spokes through TR.

The original TR-EWI therapeutic protocols available at each Hub included Amputee Care, Back Pain Clinic, Comprehensive Traumatic Brain Injury Evaluation, Home Safety Evaluation, Physical Therapy, Psychology, Speech Therapy, and Assistive Technology. Hub sites provided this broad spectrum of specialized rehabilitation services through clinical video telehealth (CVT). CVT allows a VA provider to diagnose, monitor, and treat medical conditions in real-time through interactive, secure (ie, encrypted) video technology, either from one clinic to another or directly to a Veteran patient's home. When extending TR from one clinic to another, specialized telecommunications equipment on both ends is required. Alternatively, when TR is conducted through VA Video Connect, patients use their own devices such as a smartphone, tablet, laptop, or desktop with a webcam, to securely connect via the internet to a VA provider regardless of where the Veteran or provider is located.

How therapeutic protocols have been organized and delivered in TR-EWI has depended on the requirements and structure of each clinic. ${ }^{15}$ For instance, the Amputee Care protocol is conducted in several ways. First, a team of amputation experts-usually a physician, a therapist, a prosthetist, and a rehab coordinator at the Hub-provide consults to the telehealth technician who is with the patient at the Spoke site. The team evaluates the patient with an amputation to determine the prescription for a prosthesis with the support of the technician; the prescription is then sent to a community prosthetist, and once fabricated, another virtual visit is scheduled to check that the prosthesis is meeting patient needs and complies with specifications as ordered. In a second instance, a tele-consult is made from the prosthetist at the Hub to the amputation clinic team who is working with the patient at the Spoke. A third way that this therapeutic protocol is implemented is through a provider- 
led Amputee support group for Veteran patients from Hub to Spoke.

Assistive technology via TR-EWI is another example of how specialized expertise available at the Hubs is delivered to personnel at Spokes. In this case, the Hubs provide guidance on how to best assess patients needing AT devices, such as wheelchairs and other mobility devices, sensory (eg, hearing, vision, physical) aids, and accommodations for activities of daily living. Typically, patient assessments are conducted in conjunction with the technician at the Spoke in three steps. The first session comprises the patient assessment, the second session involves the fitting of the device, and the third session is a follow-up to ensure that the device is meeting patient needs.

Additional examples of protocols provide other cases in point: For home safety assessments, an interdisciplinary rehabilitation team at the Hub uses secure video to evaluate the Veteran's home for safety hazards and assess the Veteran's functional capacity to recommend home modifications; traumatic brain injury (TBI) specialists at the Hub conduct initial TBI evaluations, follow-up visits, or family conferences through secure video with patients at VA community-based outpatient clinics (CBOCs) or at their homes; and speech language pathologists, occupational therapists or psychologists located at Hubs see patients through TR at other VA facilities or at patients' homes.

\section{Methods}

An independent team of researchers at a VA medical center in the southeast US was formed to evaluate TR-EWI as an IRB exempt clinical improvement project. The team designed a qualitative study employing the Reach, Effectiveness, Adoption, Implementation, and Maintenance Framework (RE-AIM). ${ }^{30}$ A semi-structured interview guide was developed based on guidance for approaching RE-AIM qualitatively by Holtrop, Rabin, and Glasgow (Table 1). ${ }^{31}$

A purposive sample was used to recruit key personnel involved in implementing TR-EWI at each of the four Hubs (Table 2). Ten stakeholders comprising program medical directors and program managers participated in telephone and/or video group interviews (three interviews per four sites) quarterly during the first 18 months of implementation (except at one site, for which an interview occurred at a five-month interval). Group interviews were repeated with the same individuals at all three time points except for one site where a medical director was replaced. Two other group interviews were conducted with the PM\&R program office using the interview guide.
Each group interview was co-moderated by three team members for a duration of approximately 60 minutes. Interviews were audio-recorded and hand-written notes were taken. A total of fourteen group interviews (three interviews with each of the four Hub sites plus two more with the PM\&R program office) were conducted. Handwritten notes from each co-moderator were compared to ensure consensus and notes were transcribed into an electronic format. When questions or a need for clarification arose, the audio was consulted and reheard. A list of interview responses was compiled in an excel spreadsheet for analysis within a table delineating RE-AIM domains. A final list of interview responses was compiled for coding, returning to audio recording as needed to retrieve verbatim quotes.

Constant comparison of the data to emerging conceptualizations was used throughout the analytic process. Initial analysis of the data was conducted by the first author (JLHG) and centered on the inductive identification of themes, whereby emerging conceptual categories were confirmed by other members of the research team; a subsequent analysis of the same data identified overlapping data points that consolidated themes where appropriate. Overview summaries of themes on barriers and facilitators and top key points were generated for each RE-AIM domain. A final analysis of the data and findings was conducted by a new team member with qualitative research expertise and extensive clinical rehabilitation experience $(\mathrm{CK})$ in order to assure reliability of themes developed. From this final analysis, themes were confirmed, further refined, and barriers and facilitators verified. Rigor was enhanced by researchers' ongoing interactions with study participants and member checking, the interdisciplinary nature of the study team, use of an interview guide with questions developed in alignment with the RE-AIM framework, and iterative cycles of analysis conducted by multiple researchers who have expertise in qualitative research.

\section{Results}

This study was conducted during the first 18 months of TR-EWI implementation. The data yielded three overarching themes (Table 3). The theme Cultural Factors Influencing Reach and Adoption of TR describes ways of interacting with the rehabilitation process through TR from both the Veterans' and the providers' perspectives. The Infrastructural and Logistical Factors Influencing Implementation of TR theme delineates infrastructure and logistical constraints to tele-delivery of rehabilitation. 
Table I Interview Guide Structured Using Qualitative Approach to RE-AIM Framework

\begin{tabular}{|c|c|}
\hline RE-AIM Domain and Definition Used* & Prompts and Probes \\
\hline $\begin{array}{l}\text { Reach - What factors contribute to the participation/non-participation of } \\
\text { patients in TR? What might have been done to get more patients to } \\
\text { participate in TR? }\end{array}$ & $\begin{array}{l}\text { Veterans } \\
\text { - What are barriers to serving rural Veteran patients with telerehabilitation? } \\
\text { - What are facilitators to serving rural Veteran patients with telerehabilitation? } \\
\text { - Whe specific patient profiles that are more frequently seen for telerehab? } \\
\text { - What are some reasons that Veterans prefer tele to face to face? } \\
\text { ? How many are declining the telerehab approach? } \\
\circ \text { Are you keeping track of this? } \\
\circ \text { What incentives are there to use one or the other? } \\
\circ \text { Are there any specific disincentives for the Veteran patients to use } \\
\text { telerehab? }\end{array}$ \\
\hline $\begin{array}{l}\text { Effectiveness - Is telerehabilitation working to affect the outcomes noted? } \\
\text { What other factors contribute to the results? Are the results of TR-EWI } \\
\text { meaningful and how? }\end{array}$ & $\begin{array}{l}\text { Metrics } \\
\text { - Do you have any idea on how to capture effort in telerehabilitation } \\
\text { (instead of just uniques and encounters?) } \\
\text { - How helpful is the data you are collecting now in terms of helping you } \\
\text { understand the reach of your program and how to expand that reach? } \\
\text { - What other kind of outcomes data could capture effectiveness of } \\
\text { - Welerehabilitation? } \\
\text { ism that you did not provide before? } \\
\text { - How does the tele-health delivery increase your capacity and improve your } \\
\text { quality of care? What are the new capacities that you did not have before? } \\
\text { mileage, other resources) }\end{array}$ \\
\hline $\begin{array}{l}\text { Adoption - What factors contributed to the facility and its providers taking } \\
\text { up the TR-EWI program? What barriers interacted with the program to } \\
\text { prevent adoption? Was there partial or complete adoption? Why did some } \\
\text { providers participate, and others did not? }\end{array}$ & $\begin{array}{l}\text { Sites } \\
\text { - How would you describe the adoption of tele-rehab from facility } \\
\text { leadership? } \\
\text { - What drives the adoption of the different protocols? (expertise, what } \\
\text { counts/credit, VA policy, population needs) } \\
\text { - Are there any obvious differences in the adoption of different } \\
\text { protocols? } \\
\text { - How do you recruit other facilities? } \\
\text { - How do you create partnerships outside TR-EWI and the VA? } \\
\text { - What is the minimum requirement to become a community partner? } \\
\text { Providers } \\
\text { - How do you identify providers for telerehabilitation? } \\
\text { - Who is the ideal provider for telerehabilitation? } \\
\text { - What proportion say No? } \\
\text { - How many providers are telehealth ready? } \\
\text { - How do you increase the number of tele-rehab ready providers? } \\
\text { - Wre there incentives at your facility to increase the \# of telehealth } \\
\text { - What are the barriers to becoming a telerehabilitation provider? } \\
\text { - How have the different rehabilitation specialists adapted their rehabilita- } \\
\text { tion practice to tele-delivery? } \\
\text { What are the facilitators to becoming a telerehabilitation provider? } \\
\text { delivery for rehabilitation? }\end{array}$ \\
\hline
\end{tabular}

(Continued) 
Table I (Continued).

\begin{tabular}{|c|c|}
\hline RE-AIM Domain and Definition Used* & Prompts and Probes \\
\hline $\begin{array}{l}\text { Implementation - How was TR-EWI implemented? By whom and when? } \\
\text { What influenced implementation or lack of implementation? How and why } \\
\text { was TR-EWI program adapted or modified over time? }\end{array}$ & $\begin{array}{l}\text {-What kind of guidance and how much did you receive in establishing the } \\
\text { - Wrogram? } \\
\text { - What are the barriers for implementing telerehabilitation? } \\
\text { - Describe steps required to set up a clinic. } \\
\text { - What kind of space is needed for a telerehabilitation clinic? } \\
\text { - Describe the variations and differences in implementing the different } \\
\text { - Wrotocols. } \\
\text { - Which protocols are easiest to implement? } \\
\text { - Why? } \\
\text { - Wo implement? }\end{array}$ \\
\hline $\begin{array}{l}\text { Maintenance** - Is the TR-EWI being implemented (and adapted) after the } \\
\text { implementation core period? What is sustained, what discontinued, what } \\
\text { modified- and why? }\end{array}$ & $\begin{array}{l}\text { - Please name the protocols that have been implemented thus far and state } \\
\text { whether they are at-risk or not-at-risk of being sustained into the future. } \\
\text { - Describe the sustainability plans for each. } \\
\text { ing entities? (Describe and explain reasons for anticipated changes.) } \\
\text { o Spoke sites } \\
\text { Other services within your facility } \\
\text { - Collaboration with other Hubs } \\
\text { - Collaboration with VA National Program Office } \\
\text { - Collaboration with community partners and vendors } \\
\text { Do you expect new Spokes to emerge as a product of the culture of } \\
\text { collaboration that has been fostered over TR-EWl's implementation? If so, } \\
\text { please explain. } \\
\text { - Are there other rural collaborations on your radar that you have not had } \\
\text { time to implement? } \\
\text { TR-EWI has been very successful at increasing the number of rural } \\
\text { Veterans served. Do you expect to continue to see increasing numbers } \\
\text { of rural Veterans after TR-EWl funding ends? } \\
\text { - What additional things could be done to expand reach of TR-EWI to rural } \\
\text { Veterans? } \\
\text { - How do you plan to continue tracking outcomes? }\end{array}$ \\
\hline
\end{tabular}

Notes: *All summary questions listed under RE-AIM Domain and Definitions were adapted from Holtrop, Rabin, and Glasgow. ${ }^{31}$ ***Maintenance questions were not used during the time period reported in this study.

The Rurality as a Factor in TR Implementation theme illustrates the complexity involved in extending TR to rural, low-resource environments.

\section{Cultural Factors Influencing Reach and Adoption of Telerehabilitation}

A factor frequently cited by interviewees as impacting TREWI reach and adoption of TR is cultural acceptance. Culture in TR can be defined as factors that relate to the habits, skills and practice of delivering rehabilitation remotely. ${ }^{32}$ Within VHA, TR presents providers and patients a new way of interacting with each other; TR also serves as a catalyst for changing understandings about what it means to be a rehabilitation provider as well as what it means to be a patient experiencing rehabilitation remotely. Within this theme regarding cultural factors, two subthemes were identified and are described below.

\section{Veterans' Acceptance of Telerehabilitation}

Interviewees reported only limited resistance among Veterans to replace traditional, face-to-face rehabilitation care with TR. As reported by interviewees, a minority of rural Veteran patients faced personal-level barriers to TR. Some Veterans were uncomfortable with the technologies required for rehabilitation treatments provided at a 
Table 2 Hubs and Rural Spoke Sites Used to Implement a Telerehabilitation Program for Rural Veterans

\begin{tabular}{|l|l|}
\hline Hub & Rural Spoke Site \\
\hline Richmond, Virginia & $\begin{array}{l}\text { Charlotte Hall, VA } \\
\text { Clarksburg, WV }\end{array}$ \\
\hline Minneapolis, Minnesota & $\begin{array}{l}\text { Sioux Falls, SD } \\
\text { Tomah, WI } \\
\text { Mason City, IA }\end{array}$ \\
\hline San Antonio, Texas & $\begin{array}{l}\text { Asheville, NC } \\
\text { Victoria, TX }\end{array}$ \\
\hline Seattle, Washington & Kailua Kona, HI \\
\hline
\end{tabular}

distance. Additionally, some experienced logistical challenges in engaging in the TR visit at home without the additional support of a caregiver to assist with logistics or the therapeutic activities for the sessions. TR provided both advantages and disadvantages, as described by one interviewee:

The geriatric population has its pros and cons. [TR] is difficult at times, as it is good to have a caregiver on hand for those patients ... to help them get set up and help them feel more comfortable with [the telehealth visit]. Some patients are not comfortable doing something different or new, and get anxious .... For ongoing appointments (i.e., weekly) versus a one-time thing, [older patients] who have more frequent appointments may be more open to the idea of telehealth.

Among the minority of Veteran patients who were considered more reluctant to embrace TR were those who would have to forego travel pay benefits that provide gas mileage reimbursement. Other reluctant Veterans were those who leveraged in-person appointments as opportunities to "get out of the house," as in the case of one Veteran who declined TR for the chance to combine an in-person clinic visit with a shopping trip to the city.

These exceptions apart, overall, interviewees reported high acceptance of TR among rural Veterans. Facilitators for rural Veteran use of TR centered on convenience and accessibility of appointments. Veteran patients with the most buy-in for TR were those who had ongoing or frequent appointments or who worked full time:

For [the] most part, we hear a lot of positive feedback. [TR] saves driving time, saves the Veteran time away from work when they can connect on a lunch break. Or [it helps] if there are physical reasons they can't come [in], whether it be that it's hard for them to get into the VA or to travel to the VA or if they don't have transportation to the VA.

Veterans with specific diagnoses that made entering a VHA facility challenging, such as post-traumatic stress disorder (PTSD), were said to be especially amenable to TR. TR allowed them to avoid potentially triggering situations by receiving their rehabilitation treatment at home. "[TR] also alleviates PTSD symptoms in Veterans who can connect from home. It's rare that people decline; most are open to telehealth and find it beneficial."

There was agreement among interviewees that while it may be less than optimal for Veterans to access rehabilitation sessions via the small screen of smartphones, for those who regularly use mobile phones, the use of a technology that was already embedded in their everyday lives facilitates acceptance of TR. In cases where patients had hearing or vision impairments that reduced the ability to hear or see through a device, driving impairments in these patients rendered TR as a superior option to traveling for in-person visits.

\section{Providers' Cultural Acceptance of TR}

The most frequently cited barrier for providers' adoption of TR was traditional rehabilitation cultural practice, which has typically relied on manual manipulation and face-to-face evaluations. Interviewees reported that the most common barrier against provider adoption of TR was translating what has been traditionally manual, inperson therapies into TR. Not surprisingly then, the providers who were considered most hesitant to adopt TR were chiropractors, physical therapists, occupational therapists, and kinesiologists:

[We] haven't heard much resistance in terms of "I don't like telehealth, it scares me," but I know there are people out there that think that way. We have some providers who are reluctant to use [TR] or who feel it isn't appropriate for them, for example, providers whose services are hands-on.

[For example] for the [Kinesiology Therapy] Department, as it is exercise-based, hands-on, using equipment/ machines, it's been harder to visualize how they could use it.

TR-EWI program leaders have employed several strategies to overcome barriers within the culture of rehabilitation practice. A primary facilitator for applying tele-delivery to rehabilitation practice has been TR-EWI's approach to promoting TR in an open-ended, non-prescriptive way. 
Table 3 Themes, Subthemes, and Barriers and Facilitators of Telerehabilitation (TR) Program Implementation

\begin{tabular}{|c|c|c|c|}
\hline $\begin{array}{l}\text { Themes and } \\
\text { Subthemes }\end{array}$ & Theme Description & Representative Barrier(s) & Representative Facilitator(s) \\
\hline $\begin{array}{l}\text { Cultural Factors } \\
\text { Influencing Reach and } \\
\text { Adoption of TR } \\
\text { Veterans' acceptance of } \\
\text { TR }\end{array}$ & $\begin{array}{l}\text { This theme describes the habits, } \\
\text { skills, and practices involved in TR } \\
\text { process from both Veterans' and } \\
\text { providers' perspectives. }\end{array}$ & $\begin{array}{l}\text { - Veterans' discomfort with newness of } \\
\text { using technology within rehabilitation } \\
\text { interactions } \\
\text { - Veteran forgoing mileage reimbursement }\end{array}$ & $\begin{array}{l}\text { - Caregiver availability to assist } \\
\text { with technology and/or logistics } \\
\text { - Convenience offered by TR } \\
\text { - Ability to avoid potential expo- } \\
\text { sure to triggering situations for } \\
\text { Veterans with PTSD } \\
\text { - Use of smartphones/technologies } \\
\text { that are already embedded in } \\
\text { Veterans' lives } \\
\text { - TR a benefit when Veterans are } \\
\text { challenged in driving }\end{array}$ \\
\hline $\begin{array}{l}\text { Providers' cultural } \\
\text { acceptance of TR }\end{array}$ & & $\begin{array}{l}\text { - Therapy practices that traditionally } \\
\text { rely on manual procedures }\end{array}$ & $\begin{array}{l}\text { Administrative strategies: } \\
\text { - Allowing providers autonomy and } \\
\text { flexibility in implementing TR into } \\
\text { their practice, which enabled } \\
\text { them to discover what works } \\
\text { - Program leaders' advocating for } \\
\text { TR use among providers } \\
\text { - Mentorship and development of } \\
\text { TR training in how to implement }\end{array}$ \\
\hline $\begin{array}{l}\text { Infrastructural and } \\
\text { Logistical Factors for TR } \\
\text { Implementation }\end{array}$ & $\begin{array}{l}\text { This theme describes the } \\
\text { infrastructure and logistical } \\
\text { constraints to telerehabilitation. }\end{array}$ & $\begin{array}{l}\text { - Limited space that is quiet, private, } \\
\text { ample enough for demonstrations, } \\
\text { and flexible enough to meet clinicians' } \\
\text { dynamic scheduling needs } \\
\text { - Identifying and then procuring needed } \\
\text { technology } \\
\text { - Coordination of services requiring an } \\
\text { interdisciplinary approach }\end{array}$ & $\begin{array}{l}\text { Administrative strategies: } \\
\text { - Program leaders' championing of } \\
\text { TR-related needs (eg, space, } \\
\text { equipment) } \\
\text { - Availability of personnel (ie, tech- } \\
\text { nicians) dedicated to providing } \\
\text { logistical support for the TR } \\
\text { - Development of systematic way } \\
\text { of sharing TR lessons learned }\end{array}$ \\
\hline $\begin{array}{l}\text { Rurality as a Factor in } \\
\text { TR Implementation }\end{array}$ & $\begin{array}{l}\text { This theme describes the } \\
\text { complexity involved in extending } \\
\text { TR to rural, low-resource } \\
\text { environments. }\end{array}$ & $\begin{array}{l}\text { - Complexity of providing health and } \\
\text { rehabilitation services to Veterans liv- } \\
\text { ing in rural, low-resource communities } \\
\text { - Staffing challenges; difficulty in recruit- } \\
\text { ing rehabilitation professionals to rural } \\
\text { clinics }\end{array}$ & $\begin{array}{l}\text { - When an urban care center was } \\
\text { in close enough proximity to } \\
\text { serve rural areas } \\
\text { - When there are schools and jobs } \\
\text { available in rural communities to } \\
\text { support families of rural TR } \\
\text { technicians }\end{array}$ \\
\hline
\end{tabular}

Abbreviation: PTSD, post-traumatic stress disorder.

This strategy entailed administrators' affording providers the autonomy and flexibility to incorporate telecommunications technology into their practice in ways that made the most sense for them. For some providers, they began by evaluating a patient in the clinic, where they could also provide manual treatment and training in home-exercises; this clinical strategy was then followed by a transfer of the care into the patient home assisted via tele-technology. Subsequently, with the patient at home, the provider could then monitor patient safety during prescribed exercises and observe, through the technology, the Veteran in their home to better tailor existing or additional treatments. In other situations, providers transitioned their patients to TR every other visit, whereby TR served to lighten the travel demands for the Veterans. In another example, a pre-screening for driving therapy was provided remotely before the driving rehabilitation appointment rather than requiring the Veteran to travel twice to the facility. 
Interviewees also found that TR enhanced compliance with mandatory follow-up clinics. For example, within some clinics, such as the wheelchair clinic, follow-up is required within four weeks of the Veteran receiving a wheelchair. Interviewees found that TR enabled increased compliance, which reduces risk to Veterans of health hazards due to ill-fitting chairs.

Advocating for TR use among clinical providers was another important facilitator for TR adoption. TR-EWI program leadership at Hubs championed the adoption of TR through provision of information via email listservs, presentations at staff meetings, weekly cross-service meetings with leaders in and outside of rehabilitation, and town hall discussions. In the end, TR-EWI program leaders served as de-facto telehealth champions at their respective Hubs where they encouraged and facilitated providers in and outside of rehabilitation to become telehealth ready. As champions, these leaders served as mentors to their facilities for implementing TR in partnership with other VHA services, such as primary care, as well as non-VA providers and vendors in the community.

Importantly, TR-EWI program leaders also facilitated rehabilitation practice transformation through the creation of training on how to deliver rehabilitation through telecommunications technology and adopt TR. As an illustration, for providers who had difficulty imagining how their therapies could translate into TR, one Hub launched the production of case study videos to help providers envision how rehabilitation experts could apply their therapies remotely. Through audiovisual case studies, providers were exposed to examples of therapists who transitioned their practices to TR, and where appropriate and desired by patients, gradually decreased the need for face-to-face sessions. These training tools were important for helping providers envision a new culture of rehabilitation practice. "The more providers know about [TR] and understand how they can use [TR] with their specific caseload, the more they [adopt and] promote telehealth." As described by another interviewee:

Physical Therapy (PT) is typically hands-on, so they had more difficulty seeing how they would use tele, but there has been a good response. [They are] starting to use it. Through case studies, they are starting to see how they can gradually decrease [the] amount of sessions and transfer recommendations, strategies, [and] some exercise plans into the home.

By demonstrating to providers how tele-delivery can complement or replace aspects of in-person rehabilitation care,
TR-EWI has been able to create new opportunities for increasing both access and enhancing care, even for therapies that are not typically amenable to TR.

\section{Infrastructural and Logistical Factors for TR Implementation}

Procuring appropriate equipment and having enough bandwidth capacity in the network were recurrent barriers to TR implementation. Additionally, finding appropriate space in already space-limited facilities was also identified as a recurring barrier for TR. For example, provider space for TR needs to be insulated from background noise that can affect the quality of the video call:

Space can still be an issue if you don't have the private area or private clinic space to conduct your visit. You need a quieter space versus, you know, like an open clinic that can be harder to conduct the visit in.

Just as importantly, the space must be ample enough for the therapist to conduct treatment; for example, to demonstrate how to do a prescribed exercise requires enough space for the camera to capture the full body of the therapist.

Interviewees also noted that providing space to parttime therapists or for ad-hoc treatments was a logistical challenge. This has meant coordinating and managing a shared space, which adds another logistical barrier in the form of management and efficient allocation of that space.

As one interviewee noted:

Most of the providers are not going to become full-time tele-rehab providers, so it is challenging to get space and equipment that can be used on a rotating basis. And [the question remains] who is going to manage that space?

Logistical issues were also important to resolve in TR implementation. Some therapeutic protocols, such as assistive technology, required an interdisciplinary approach and the coordination of multiple specialists. As one interviewee explained:

Assistive technology is more complex by nature so we're pursuing an interdisciplinary assistive technology wheelchair clinic involving the physician, the PT, the OT and ... our rehab engineer who specializes in assistive technology. So, right now we've been conducting weekly meetings to try to set this clinic up, but it's heavy on the administrative needs to ... process through all that is required due to the complexity of this clinic. 
Implementing TR without the requisite infrastructure and logistical resources, could be, as stated by one interviewee, "very discouraging to providers." Interviewees suggested that if obstacles remained to these basic requisites for TR, providers could be so discouraged as to be unlikely to try tele-delivery of their therapies again, thus abandoning TR.

Several facilitators were identified as contributing to the uptake of TR. Overwhelmingly, interviewees discussed the importance of site champions and hospital leadership support. Interviewees reported that procuring dedicated clinic space and equipment for TR, and efficient scheduling mechanisms, were primarily mitigated through the perseverance of site champions and buy-in from facility leadership. Moreover, when facilities had a telehealth coordinator who was well integrated into the range of hospital and clinic services, these individuals were instrumental in working with TR-EWI program leaders in creating TR clinics and integrating TR logistical needs into the facility's existing processes. Additionally, when clinical technicians who could be dedicated to telehealth were available, these personnel were also described as vital for coordinating TR space and scheduling:

Everything that [we've been] doing takes a lot of time to coordinate and process. It includes multiple team members from different areas so a good working relationship with everybody is important.

As a final facilitator, the development of a systematic way of sharing TR lessons learned was described as fundamental to resolving challenges to TR implementation. From the launch of the program and throughout its implementation, TR-EWI program leaders prioritized the frequent sharing of lessons learned. This was done through Hub and Spoke monthly, quarterly, and annual meetings at all sites. This continuous sharing of lessons learned allowed for a more rapid and synergistic deployment of TR through the program. This strategy also served to maximize the exchange of experience and ideas across sites, which created a community of practice that could leverage for the VHA a collective wealth of experience.

\section{Rurality as a Factor in TR Implementation}

One theme that was extensively reported on was the complexity of extending services to rural patients. This complexity served as a major barrier to TR implementation in rural settings. While rural Veterans participating in TREWI reported a high degree of satisfaction with TR (paper forthcoming), rurality impacted TR implementation in several ways. For TR that was delivered to Veterans' homes, a lack of stable broadband and poor connectivity could generate frustration and interfere with successful rehabilitation treatment for patients. Additionally, interviewees noted that for rural Veterans with complex health problems in low-resource environments, the services patients' needs are often the ones that are the most difficult to provide.

One example discussed involved the fitting of wheelchairs through the assistive technology clinic. While rehabilitation expertise was available at the Hub, its implementation still required hands-on fitting, assessment, measurement, trial, equipment, and transfers into a wheelchair; the need for skilled hands-on assistance complicated these types of tele-rehabilitation consults. While having a person on-location in the rural community was necessary for such TR, it posed real challenges; as one program manager asked rhetorically, "Where do you get a [skilled hands-on] person if the Veteran lives four hours away?"

Vendors in the community could be an option, but there are questions to consider such as if a vendor can accomplish the task at a reasonable price. Alternatively, a telehealth technician at [a] VA [facility] could be trained to do the kinds of highly-skilled tasks needed for fitting assistive technology, but to do this would require [the technician] to travel long distances with the equipment: you need a big van, a ramp, etc., and a lot of time too ... for a single Veteran that lives very far away.

The lack of an existing network of rehabilitation support for rural Veterans is exacerbated by geographical distances as well as the resource limitations that are characteristic of rural communities. This makes reaching the most rural Veterans where they live far more challenging. As one interviewee pointed out, even when there is a plan to provide services, environmental limitations can impede the execution of the plan:

Things can go off-track very fast. The resources become a significant barrier the more rural you get, resulting in a situation where Veterans who can be more readily served are those who are not as far away.

Another commonly cited barrier for rural TR are staffing challenges in rural communities. Although approved Spokes received funding support, not all Spokes could make use of the funding to hire personnel for TR in rural areas. In the case of one Hub, recruiting a telehealth technician for their Spoke proved insurmountable due to 
the region's lack of schools, housing, and job opportunities for trailing spouses:

If you are prioritizing highly rural areas then almost by definition, those are not the areas that have schools that are good for young families, or that a spouse can come and easily find a job.

Choosing appropriate Spokes in rural areas thus entails what one interviewee referred to as "the goldilocks phenomenon"- the setting chosen must be close enough to an urban area to remain viable for staffing but far enough that it serves a rural population. To facilitate implementation of TR for rural Veterans, the Hub discussed above overcame staffing barriers by changing its Spoke to another site that was less rural and had a pre-existing telehealth technician.

Overall, interviewees expressed that the nature of lowresource environments entails more work to serve fewer people. One interviewee emphasized that while "best practices" is a current buzzword, these are often imagined in the context of ample resources, readily accessible equipment, and plentiful skilled personnel, which is far from a reality for most rural areas:

When we consider what we want to do, we're wishful, and we are hopeful that we can do as much as can. But when we think about what is within our means, it is improving practice, not necessarily best practice, that we can do.

\section{Discussion}

This study identifies and explicates the most salient barriers and facilitators encountered during the first 18 months of implementation of a TR program within a national healthcare system in the United States. It contributes new and indepth understanding of the processes by which TR is implemented in a large healthcare system. The main goal of TREWI has been to increase rehabilitation access and enhance rehabilitation quality to provide the best care to rural Veterans. By inquiring into factors impacting TR for rural Veterans, this study suggests that TR-EWI has successfully applied creative approaches to addressing the many complex barriers that accompany translating rehabilitation into tele-delivery for rural patients.

Most notably, this study contributes nuanced understanding of cultural barriers to TR reach and adoption. Barriers were found at both the Veteran level and the level of the rehabilitation provider. While cultural barriers are generally some of the most challenging barriers to overcome, ${ }^{33}$ study findings indicate that, at both the clinician and the patient level, TR-EWI has begun to foster a transformation in the cultural practice of rehabilitation.

In our study, logistical constraints to TR, such as space, equipment, and scheduling considerations, served to challenge TR's implementation. Procuring appropriate equipment was also a recurrent barrier to TR implementation. Just adding a camera to an existing workstation is not enough to optimize TR. For TR, a one-size-fits-all approach does not always work and a range of options is needed depending on the specific therapy. On the providers' end, there are portable units and dedicated units; having a portable unit is advantageous, especially if an unanticipated video consult is needed. Moreover, while a rehabilitation counseling session could be successful using basic video teleconferencing equipment, fitting a prosthetic device would need to capture different dimensions to show the patient how to use the device appropriately. TR equipment needs to be adaptable to different scenarios.

Findings regarding infrastructural barriers to rural care, specifically lack of broadband, are consistent with published research regarding telehealth in rural areas. ${ }^{34}$ As such, extending the reach of TR to Veterans who reside in rural, low resource environments entails finding solutions to infrastructure. Implementing TR to meet the rehabilitation needs of Veterans in resource-limited rural environments requires creative approaches and flexibility. This, in combination with logistical constraints to TR, adds greater complexity to meeting the needs of rural patients.

We thus found that rurality brings its own unique set of challenges that are important considerations in extending TR to Veterans living in rural locations. Study findings suggest that rurality may be worth considering as a type of patient complexity that may need to be accounted for when treating rural patients; similar to the ways that comorbidities are taken into consideration when appraising a patient's complexity.

A strength of this study is the novel use of the RE-AIM framework for guiding qualitative inquiry into TR implementation. The RE-AIM framework was originally developed to improve reporting of health promotion and healthcare research findings and facilitate their translation into real-world settings; ${ }^{35}$ however, the qualitative application of RE-AIM has been less frequent in the health services research. ${ }^{36}$ Consequently, this study is novel in two ways. First, we developed our qualitative interview guide based on RE-AIM domains, which gave structure to an interview process that was conducted in three phases over the first 18 months of TR-EWI implementation. The 
RE-AIM structure served as a useful framework for adjusting the focus of the interviews in a way that allowed continuity of the sequential interviews over time. This structure allowed for flexibility in the interviews' foci as implementation of the TR-EWI program evolved over time. Secondly, we applied RE-AIM to evaluate the implementation of a care delivery system, which was a different application from how RE-AIM is typically applied to develop and test interventions. ${ }^{37}$ We found that applying RE-AIM pragmatically ${ }^{37}$ was useful in guiding our qualitative evaluation of the barriers and facilitators to TR.

Our use of qualitative thematic analysis, in combination with the small sample size, limits the generalizability of study findings. However, the sample of program managers and medical directors, by virtue of their vantage as administrators, were able to speak to the process of program implementation with depth and nuanced understanding of both practitioner and administrative concerns. As such, our interviewees provided meaningful insight into the complexities of implementing a TR program to individuals residing in rural locations. This study contributes indepth knowledge regarding the process of providing TR for rural Veterans, and most notably, points to practical real-world lessons with implications for how TR can be implemented and sustained into the future.

\section{Acknowledgments}

We acknowledge Jessica Barton for her thoughtful review of the revised manuscript. We would also like to recognize the outstanding editorial support of Lynn Dirk, MAMC, through affiliation of the North Florida/South Georgia Veterans Health System with the North Florida Foundation for Research and Education, Inc. Funding was provided by the Office of Rural Health, U.S. Department of Veterans Affairs.

\section{Disclaimer}

The opinions expressed herein are those of the authors and do not necessarily reflect those of the US Department of Veterans Affairs, or any of its affiliated institutions.

\section{Disclosure}

The authors report no conflicts of interest in this work.

\section{References}

1. Offner R, Seekins T, Clark F. Disability and rural independent living: setting an agenda for rural rehabilitation. Human Serv Rural Environ. 1992;15:6-8.
2. Dew A, Bulkeley K, Veitch C, et al. Addressing the barriers to accessing therapy services in rural and remote areas. Disabil Rehabil. 2013;35(18):1564-1570. doi:10.3109/09638288.2012.720 346

3. Echeverri RC Barriers to participation in cardiac rehabilitation: a rural perspective. 2007.

4. Schmeler MR, Schein RM, McCue M, Betz K. Telerehabilitation clinical and vocational applications for assistive technology: research, opportunities, and challenges. Int $J$ Telerehabil. 2009;1:59-72. doi:10.5195/IJT.2009.6014

5. Randall M, Kilpatrick KE, Pendergast JF, Jones KR, Vogel WB. Differences in patient characteristics between veterans administration and community hospitals: implications for VA planning. Med Care. 1987;25(11):1099-1104. doi:10.1097/00005650-198711000-00008

6. U.S. Department of Veterans Affairs. Office of Rural Health. 2019. Available from: https://www.ruralhealth.va.gov/aboutus/ruralvets.asp. Accessed January 11, 2020.

7. Hale-Gallardo J, Jia H, Delisle T, et al. Enhancing health and independent living for veterans with disabilities by leveraging community-based resources. $J$ Multidiscip Healthc. 2017;10:41-47. doi:10.2147/JMDH.S118706

8. Shannon RH, Maloney DL. Telemedicine at the veterans health administration. Comp Large Int Health Net. 1997;447-465.

9. Nickelson DW. Telehealth and the evolving health care system: strategic opportunities for professional psychology. Prof Psychol Res Pract. 1998;29:527. doi:10.1037/0735-7028.29.6.527

10. Hatzakis M, Haselkorn J, Williams R, Turner A, Nichol P. Telemedicine and the delivery of health services to veterans with multiple sclerosis. J Rehabil Res Dev. 2003;40(3):265-282.

11. Bendixen RM, Levy C, Lutz BJ, Horn KR, Chronister K, Mann WC. A telerehabilitation model for victims of polytrauma. Rehabil Nurs. 2008;33(5):215-220. doi:10.1002/j.2048-7940.2008.tb00230.x

12. Bendixen RM, Levy CE, Olive ES, Kobb RF, Mann WC. Cost effectiveness of a telerehabilitation program to support chronically ill and disabled elders in their homes. Telemed J E Health. 2009;15 (1):31-38. doi:10.1089/tmj.2008.0046

13. Cherry COB, Chumbler NR, Richards K, et al. Expanding stroke telerehabilitation services to rural veterans: a qualitative study on patient experiences using the robotic stroke therapy delivery and monitoring system program. Disabil Rehabil Assist Technol. 2017;12(1):21-27. doi:10.3109/17483107.2015.1061613

14. Housley SN, Garlow AR, Ducote K, et al. Increasing access to cost effective home-based rehabilitation for rural veteran stroke survivors. Austin J Cerebrovasc Dis Stroke. 2016;3:1-11.

15. Scholten J, Poorman C, Culver L, Webster JB. Department of veterans affairs polytrauma telerehabilitation: twenty-first century care. Phys Med Rehabil Clin N Am. 2019;30:207-215. doi:10.1016/j. pmr.2018.08.003

16. Harada ND, Dhanani S, Elrod M, Hahn T, Kleinman L, Fang M. Feasibility study of home telerehabilitation for physically inactive veterans. J Rehabil Res Dev. 2010;47.

17. Brennan DM, Mawson S, Brownsell S. Telerehabilitation: enabling the remote delivery of healthcare, rehabilitation, and self management. In: Gaggioli A, Keshner EA, Weiss PL, Riva G, editors Advanced Technologies in Rehabilitation: Empowering Cognitive, Physical, Social and Communicative Skills Through Virtual Reality, Robots, Wearable Systems and Brain-Computer Interfaces. Amersterdam: IOS Press BV; 2008

18. Peretti A, Amenta F, Tayebati SK, Nittari G, Mahdi SS. Telerehabilitation: review of the state-of-the-art and areas of application. JMIR Rehabil Assist Technol. 2017;4:e7. doi:10.2196/rehab. 7511

19. Brienza DM, McCue M. Introduction to telerehabilitation. In: Kumar S, Cohn ER, editors. Telerehabilitation. London: Springer; 2013:1-11. 
20. Chumbler NR, Quigley P, Sanford J, et al. Implementing telerehabilitation research for stroke rehabilitation with community dwelling veterans: lessons learned. Int J Telerehabil. 2010;2:15-22. doi:10.51 95/IJT.2010.6047

21. McCue M, Fairman A, Pramuka M. Enhancing quality of life through telerehabilitation. Phys Med Rehabil Clin N Am. 2010;21:195-205. doi:10.1016/j.pmr.2009.07.005

22. Schein RM, Schmeler MR, Brienza D, Saptono A, Parmanto B. Development of a service delivery protocol used for remote wheelchair consultation via telerehabilitation. Telemed J E Health. 2008;14 (9):932-938. doi:10.1089/tmj.2008.0010

23. Jarvis-Selinger S, Chan E, Payne R, Plohman K, Ho K. Clinical telehealth across the disciplines: lessons learned. Telemed $J E$ Health. 2008;14(7):720-725. doi:10.1089/tmj.2007.0108

24. Pramuka M, van Roosmalen L. Telerehabilitation technologies: accessibility and usability. Int $J$ Telerehabil. 2009;1:85-98. doi:10. 5195/IJT.2009.6016

25. Ackerman MJ, Filart R, Burgess LP, Lee I, Poropatich RK. Developing next-generation telehealth tools and technologies: patients, systems, and data perspectives. Telemed $J$ E Health. 2010;16(1):93-95. doi:10.1089/tmj.2009.0153

26. Kairy D, Messier F, Zidarov D, et al. Evaluating the implementation process of a new telerehabilitation modality in three rehabilitation settings using the normalization process theory: study protocol. Int $J$ Healthcare Manag. 2017;1-8.

27. Cary MP Jr, Spencer M, Carroll A, et al. Benefits and challenges of delivering tele-rehabilitation services to rural veterans. Home Healthc Now. 2016;34(8):440-446. doi:10.1097/NHH.0000000000000441

28. Gagnon M-P, Duplantie J, Fortin J-P, Landry R. Implementing telehealth to support medical practice in rural/remote regions: what are the conditions for success? Implement Sci. 2006;1:18. doi:10.1186/ 1748-5908-1-18
29. Castaneda G, Cowper Ripley D, Jia H, et al. Tracking the impact of the office of rural health $(\mathrm{ORH})$ tele-rehabilitation enterprise wide initiative by rurality fy 2017 - fy 2019. ISPRM 2020 World Congress and Association of Academic Physiatrists (AAP) Annual Meeting. 2020 .

30. Glasgow RE, Vogt TM, Boles SM. Evaluating the public health impact of health promotion interventions: the re-aim framework. Am J Public Health. 1999;89(9):1322-1327.

31. Holtrop JS, Rabin BA, Glasgow RE. Qualitative approaches to use of the re-aim framework: rationale and methods. BMC Health Serv Res. 2018;18(1):177. doi:10.1186/s12913-018-2938-8

32. Swidler A. Culture in action: symbols and strategies. Am Sociol Rev. 1986;51(2):273-286. doi:10.2307/2095521

33. McClellan MJ, Florell D, Palmer J, Kidder C. Clinician telehealth attitudes in a rural community mental health center setting. J Rural Ment Health. 2020;44(1):62-73. doi:10.1037/rmh0000127

34. Douthit N, Kiv S, Dwolatzky T, Biswas S. Exposing some important barriers to health care access in the rural USA. Public Health. 2015;129(6):611-620. doi:10.1016/j.puhe.2015.04.001

35. Glasgow RE, Vogt TM, Boles SM. Evaluating the public health impact of health promotion interventions: the re-aim framework. Am J Public Health. 1999;89:1322-1327.

36. Gaglio B, Shoup JA, Glasgow RE. The re-aim framework: a systematic review of use over time. Am J Public Health. 2013;103(6): e38-e46. doi:10.2105/AJPH.2013.301299

37. Glasgow RE, Estabrooks PE. Pragmatic applications of re-aim for health care initiatives in community and clinical settings. Prev Chronic Dis. 2018;15:E02-E02. doi:10.5888/pcd15.170271
Journal of Multidisciplinary Healthcare

\section{Publish your work in this journal}

The Journal of Multidisciplinary Healthcare is an international, peerreviewed open-access journal that aims to represent and publish research in healthcare areas delivered by practitioners of different disciplines. This includes studies and reviews conducted by multidisciplinary teams as well as research which evaluates the results or conduct of such teams or healthcare processes in general. The journal

\section{Dovepress}

covers a very wide range of areas and welcomes submissions from practitioners at all levels, from all over the world. The manuscript management system is completely online and includes a very quick and fair peer-review system. Visit http://www.dovepress.com/testimonials. php to read real quotes from published authors. 